\title{
Rover mobility on granular soil: Marrying multi-scale modeling and high fidelity experiments to infer soil stresses under the moving wheel
}

\author{
I. Vlahinic ${ }^{1}$, J. Andrade ${ }^{1}$, K. Skonieczny ${ }^{2}$, S. Moreland ${ }^{2}$, and D. Wettergreen ${ }^{2}$ \\ ${ }^{1}$ Department of Mechanical and Civil Engineering, California Institute of Technology, \\ 1200 E. California Blvd., Pasadena, CA 91127; ph (626) 395-4141; \\ email: ivan@caltech.edu; jandrade@caltech.edu. \\ ${ }^{2}$ Robotics Institute, Carnegie Mellon University, 5000 Forbes Avenue, Pittsburg, PA \\ 15213; ph (412) 268-5421; email: kskoniec@ andrew.cmu.edu; smoreland@ @ cmu.edu; \\ dsw@ri.cmu.edu.
}

\begin{abstract}
In this work, we show how high resolution imaging coupled with a novel physics-based computational framework can provide a rich description of regolithstructure interactions, using the rover mobility in soft soil as a challenging canonical problem. The images of soil deformation under a rolling wheel were collected at $7 \mathrm{~Hz}$ resolution in time and $0.19 \mathrm{~mm}$ per pixel in space, with the camera approximately following the wheel motion. The soil under the wheel is a lunar simulant GRC-1. Image correlation (optical flow) was used to infer material velocities, i.e. regolith kinematics, at near-grain scale. From this data, strains can be calculated during postprocessing, but stresses are beyond the reach of experimental measurements.
\end{abstract}

Here we present a method to infer material stresses under the rolling wheel using a multiscale framework with a simple Drucker-Prager material description. From a purely computational standpoint, this mobility problem is extremely challenging involving wheel-regolith contact and interacting localization bands on the material scale, as indicated by the experiments. Capturing these features with the correct constitutive description is at the very cutting edge of computational research today. We bypass some of the aforementioned difficulties by inferring from experimental images a key plastic internal variable, the dilatancy, which is known to control strength and softening in dilative granular materials, and use its evolution directly in multi-scale computations. The method successfully marries experiments and computations in order to quantify material stresses under the wheel, which at present are beyond the reach of either method alone. From a practical standpoint, an understanding of the material stress state is helpful for several reasons, perhaps the chief of which is that stress distributions serve as input into reduced-order mobility models, e.g. Bekker-Wong terramechanics expressions.

\section{EXPERIMENTAL BACKGROUND}

Many problems in engineering design require a thorough understanding of interactions between some type of structure or a structural element, and the ground or soil. From a computational perspective, among the most challenging problems are the soil-structure interaction problems involving granular soils, owing to this material's 
history-dependent response, fluid-like rheology at critical state, and material softening.

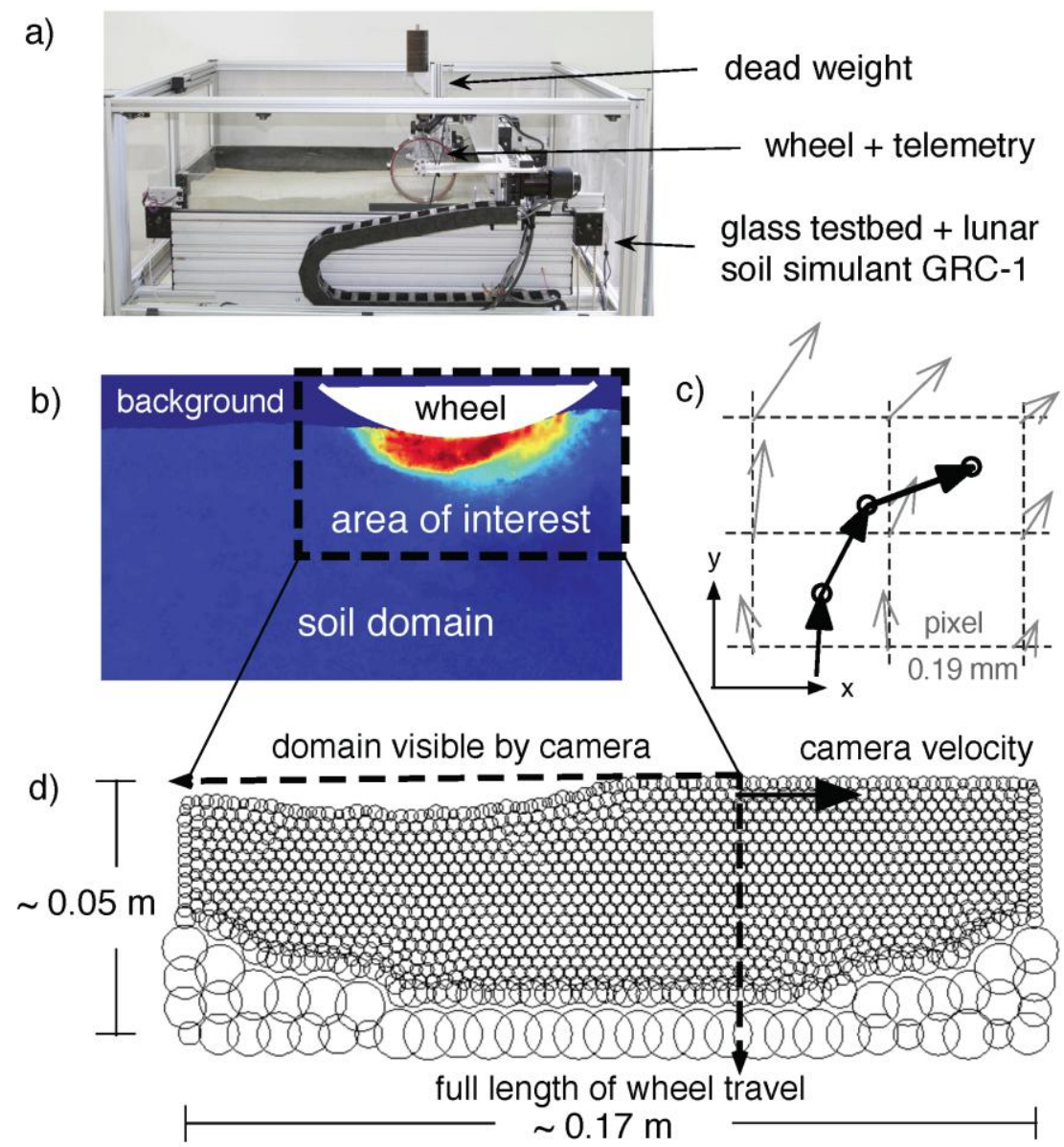

Figure 1: Experimental testbed and post-processing steps used to develop the computational mesh. (a) As the wheel travels from left to right, the camera follows the wheel motion and collects images of soil where it interfaces with the wheel. Via the optical flow algorithm during post-processing, the experiment furnishes the velocity vectors at each imaged pixel, the magnitude of which is shown in image of Fig. 1b. (b) A digital image showing magnitudes of velocity vectors at time station $t=0$. Relevant areas of the image, i.e. the background, wheel, and soil, are indicated on the image. (c) Method of interpolating the motion of Lagrange tracers in a fixed Eulerian grid, using bi-linear shape functions and nodal velocities. (d) A computational mesh is created for the entire length of wheel travel (from $t=0$ to $t=7$ sec) for a subdomain of interest. The center of each circle shown represents the nodal location of the finite element mesh. The average motion of Lagrange tracers inside each circular area represents the displacement of the center node.

In this work, we examine a canonical problem: a small rover wheel rolling across a fine granular regolith, with the intent to quantify the response of soil under the wheel. The testing was performed under the auspices of a lunar exploration program. The regolith used in experiments was the Glenn Research Center lunar simulant (GRC-1). 
The experimental apparatus is shown in Figure 1a, and consists of a glass-walled soil bin, a lunar simulant, a wheel module with a horizontally actuated motion control, and a camera that roughly follows the wheel motion and collects images of soil where it interfaces with the wheel. The experimental setup is designed to simulate a full wheel in unconstrained boundary conditions, utilizing a centerline plane of symmetry. The contact surface between the half-wheel and the glass-walled bin supplies minimal friction.

The key pieces of information gathered from experiments are the grain-scale images of GRC-1 soil as the wheel slowly travels across its surface. A $50 \mathrm{~mm}$ macro lens mounted on a digital SLR camera body was used for recording. The images were collected at a resolution of approximately 7 frames per second in time and $0.19 \mathrm{~mm}$ per pixel in space and were processed using optical flow technique via an algorithm presented by Black \& Anandan (1996). The algorithm uses clustering in order to calculate the displacement fields between pixels in successive frames. This furnishes the soil velocity vectors at each pixel and time interval, i.e. the near grain-scale soil kinematics during wheel motion.

\section{FROM EULERIAN KINEMATICS TO LAGRANGIAN FE MESH}

We recall that experiments furnish images that are taken with a camera that follows the wheel motion. Compared to a stationary camera, a follower-camera improves the resolution of the wheel-soil interface because it captures only the most relevant parts of the domain. This is particularly helpful for example when the experiment involves several rotations of the wheel. A drawback is that the soil velocity vectors or soil kinematics inferred from a follower-camera are of Eulerian type and must be converted into the Lagrangian frame of reference prior to computational analysis. This is because granular materials are history-dependent and the variables with which one describes their constitutive response (the relation between the stress and strain) are defined with respect to the reference state of the material. To accomplish the conversion several post-processing need to be performed. Ultimately, the information gathered at this stage will be sufficient to prepare the finite element mesh and the displacement boundary conditions needed for computing the soil stresses:

\section{Follow Lagrangian tracers in a fixed Eulerian grid}

In this work, the Eulerian grid is $750 \times 1500$ pixels in size, with an initial tracer placed at a center point of each pixel, for a total of approximately 1.16 million tracers. The size of the domain corresponds to the total pixel area that the digital camera "sees" during the entire experiment (recall that the camera is not stationary). The velocity of each tracer, and thus its location at the next time step, is interpolated from the experimental soil velocity vectors after the motion of the camera has been subtracted, via procedure schematically shown in Figure 1c using standard bilinear shape functions. 


\section{Clean up tracers at solid/wheel/background interfaces}

For each experimental image frame, the pixels belonging to the soil domain are delineated from the wheel/background pixels (Fig. 1b). In time, some Lagrange tracers may migrate from the soil domain in one frame to the wheel/background region in the next frame, or vice versa. Tracers that do not remain within the soil domain during the entire experiment are discarded, here accounting for less than $1 \%$ of all tracers.

III. Mesh the domain and assign nodal degrees of freedom per experiment

From the imaged soil domain, a subdomain is selected so that the wheel-soil interaction could be studied in closer detail. The subdomain is meshed using the triangular elements, via a meshing technique described by Persson \& Strang (2004). The greatest mesh density is used in the regions of most interest. Nodal displacements are assigned by averaging the displacements of all tracers within a prescribed radius from a given nodal location (Fig. 1d). The initial node-tracer associations were determined at time $\mathrm{t}=0$ and were maintained for the entire duration of the experiment.

\section{EXPERIMENTS AND THE MULTI-SCALE COMPUTATIONAL FRAMEWORK}

At the heart of any a finite element method (FEM) is a material subroutine, where the constitutive response of a given material is defined, e.g. GRC-1 in the current canonical problem. The constitutive response of granular materials is usually pathdependent and non-linear, necessitating that the finite element procedure that is incremental and iterative. But what should be the constitutive model for complex granular materials, such as the lunar regolith or its simulant? If the material kinematics are available at the lower scales, as furnished by experiments described in this work for example, how can one use this additional information to develop better computational models?

Here we describe a multi-scale method that can bypass the need for phenomenology in granular materials (Andrade and Tu, 2009). As an example, we utilize the DruckerPrager (DP) material model, although other more general models could be used as well, e.g. Matsuoka-Nakai which is a smooth version of the classic Mohr-Coulomb model. Within the DP framework, the key variable controlling the strength development and hardening/softening of granular materials is the dilatancy. An elasto-plastic recipe for a DP model is shown below, where ${ }_{v}^{p},{ }_{s}^{p}$ and $p, q$ are the volumetric and deviatoric invariants of the plastic strain and stress. Physically, is

$$
\begin{aligned}
& F(,)=q+p \quad c \quad \text { (yield function) } \\
& G(,)=q+p \quad \text { (plastic potential) } \\
& p=\operatorname{tr}(\quad), q=\sqrt{3 / 2}\|s\|, s=\quad p \quad \text { (stress invariants) } \\
& ={ }_{v}^{p} /{ }_{s}^{p}, \quad={ }_{o}+\quad \text { (plastic internal variables) }
\end{aligned}
$$




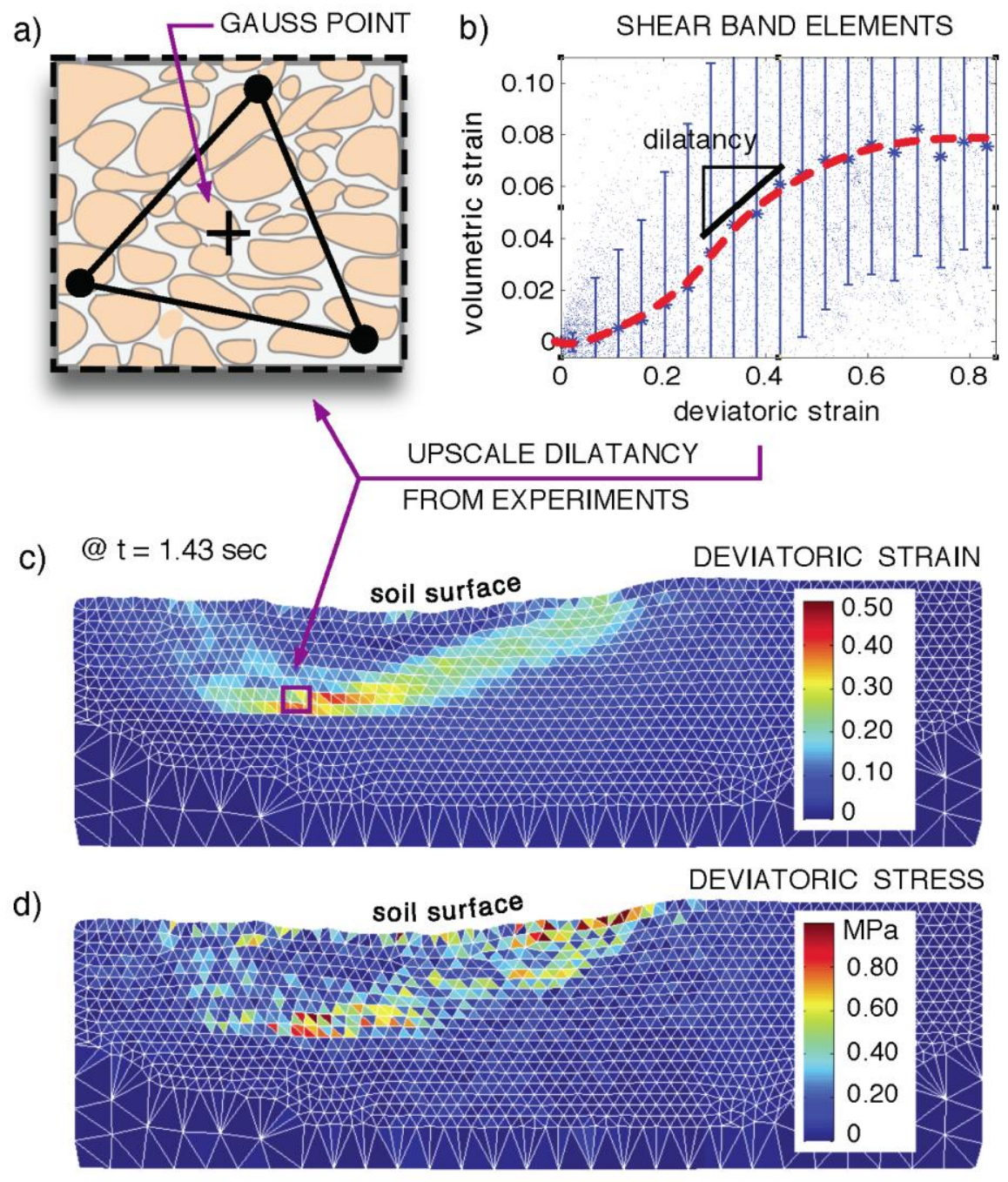

Figure 2: Computational recipe used to infer soil stresses under the moving wheel. (a) Continuum representation of a true discontinuous material response using the triangular constant-strain finite elements. The relative grain size of GRC-1 is exaggerated in comparison to the finite element size. (b) Relationship between the volumetric and the deviatoric strains inside the 200+ shear band elements as inferred from experimental data (50 time steps, for a total of $1000+$ data points). While the statistical scatter of values is significant, as indicated by the vertical one-standard-deviation error bars with fine data points shown in the background, the statistical mean (blue stars) clearly shows a dilative tendency of even an 'initially loose' GRC-1. Dashed red line is a visual fit through the mean values. (c) Total deviatoric strain after 10 camera frames of motion $(\sim 1.43 \mathrm{sec})$. Note: Wheel location can be inferred from the soil surface topography and by comparison with Fig. 1b. (d) Calculated deviatoric stress at the same time station, using a Drucker-Prager constitutive model and experimental dilatancy values in Fig. 2 b.

the friction angle, which at yield is equal to $=q / p$. The dilatancy, , captures the tendency of the granular materials to change volume under deviatoric or shear loading. The values of dilatancy inferred from experimental data are shown in Fig. $2 \mathrm{~b}$. Total friction, , is classically assumed to be the sum of the friction at critical state, 
${ }_{o}$ (typically constant during quasi-static loading), and the dilatancy, , i.e. $={ }_{o}+$.

\section{RESULTS AND OUTLOOK}

Some preliminary results are shown in Figs. $2 \mathrm{c}$ and $2 \mathrm{~d}$. The material parameters other than the experimental dilatancy values (elastic moduli and the critical-state friction) are assumed constant and were inferred from the recent characterization of GRC-1 by Oravec et al. (2010). In order to alleviate problems with localization inside the soil domain, in addition to standard boundary conditions, we also prescribe nodal displacement at critical parts of the soil domain. Efforts are ongoing to include nonlocal descriptions of material failure in order to make the computational recipe more robust.

We have presented a preliminary effort that synergizes experimental and computational methods to produce something more than the methods are capable of producing individually. Specifically, we have calculated stress profiles under the moving wheel, while avoiding the need to resort to phenomenological description of granular materials. The immediate application of the presented work is in the terms of mobility of lightweight vehicles, e.g. rovers in soft terrain. Classical terramechanics methods were developed with large, heavy vehicles in mind and often fail to agree with experimental data when scaled down to locomotion of small, lightweight vehicles. The applicability of the method should also be extendable to other types of soil-structure interaction problems, e.g. soil excavation.

\section{REFERENCES}

Andrade, J. E. and Tu, X. (2009) "Multiscale framework for behavior prediction in granular media", Mech. Mat. 63(1) 75-104.

Black, M. J. and Anandan, P. (1996) "The robust estimation of multiple motions: Parametric and piecewise-smooth flow fields", CVIU 63(1) 75-104.

Oravec, H. A., Zeng, X., and Asnani, V. M. (2010) "Design and characterization of GRC-1: A soil for lunar terramechanics testing in Earth-ambient conditions", J. Terramech. 47(6) 361-377.

Persson, P.-O. and Strang, G. (2004) "A simple mesh generator in MATLAB", SIAM review 46(2) 329-345. 